\title{
ORGANICITY: LESSONS FROM AN EXPERIMENTATION AS A SERVICE MODEL FOR DIGITAL CIVIC INNOVATION
}

\author{
D. Wilson ${ }^{1 *}$, S. McLoughlin ${ }^{2}$ and M. Brynskov ${ }^{3}$ \\ ${ }^{1}$ University College London, London, UK \\ ${ }^{2}$ LERO, Maynooth University, Maynooth, Ireland \\ ${ }^{3}$ Aarhus University, Aarhus, Denmark \\ * Corresponding author
}

\begin{abstract}
In the paradigm of the Smart City, cities are embracing new digital technologies and data innovation to redefine their relationship to citizens and enterprise. Increasingly, cities are developing visions, strategies, and related digital masterplans and action groups with which to coordinate these efforts. The European Horizon 2020 funded OrganiCity project explored a new model for providing access to all citizens to collaboratively develop and test their ideas for managing and improving the urban environment using data. The people centred and data driven approach of the OrganiCity project developed an Experimentation as a Service (EaaS) model across 13 cities and with 43 experiments. In this paper, we describe the 4 key service pillars that emerged through designing a platform to enable experimentation and the associated engagement practices required to facilitate testing in a city. The service pillars are: systematic experimentation, co-creation, federated ethics \& privacy, and management of liability \& Intellectual Property Rights. The EaaS approach provided a low-risk service blueprint for city authorities to democratically source, test and support scaling-up innovative solutions to their city challenges. Analysis of experimenter's projects highlighted the importance of shared infrastructure for reducing the barrier to entry for accessing the digital tools, but more importantly highlighted the investment required, and value of, the human resources required to facilitate the process of experimentation.
\end{abstract}

\section{Introduction}

A core focus in the emerging paradigm of 'Smart Cities' is the propositioning of urban (big) data focused solutions as a response to pressing city challenges, and digital transformation imperatives (McLoughlin et al., 2018). Many city digital strategies currently state the priority of developing urban Data / IoT (Internet of Things) use-cases as a precursor to realising urban data ecosystems and city data marketplaces that serve citizens and support enterprise. Here we follow McLoughlin et al. (2019) by defining 'urban' as 'relating to a town or city' and 'Urban Data' as data concerning a town or city physical, sociocultural, political or economic environment. Taking the example of London's Data Strategy (GLA, 2016), it premises that, "data offers new insights and ways of delivering better services to citizens - from public health...to retail in the private sector"... whereby "the city needs to be planned and built with data, and future data exploitation, in mind". Indeed, over half of Londoners using public transport in the city are using one of the 600 apps now being powered specifically using Transport for London's open data (TfL, 2017). Importantly, many such strategies support a Living Lab approach to developing innovative services, acknowledged as "a catalyst of growth and competitiveness" (Coenen et al., 2014) and have become commonplace in European research collaborations (Dutilleul et al., 2010, McLoughlin et al, 2018, Schuurman et al., 2015). The key characteristics of Living Labs as an approach, methodology or facility for innovation, prioritises co-creation with an emphasis on citizen engagement and real-world experimentation in the developing and testing of artefacts (McLoughlin et al., 2018). The OrganiCity project described in this paper builds on similar motivations of encouraging open innovation (Chesbrough et al., 2006) and a quadruple helix approach (Curley \& Salmelin, 2018) to support the challenges associated with implementing complex technology-based systems in the very human environment of the city (Wilson, 2018).

The OrganiCity ${ }^{1}$ project was envisioned as an evolving prototype of an Experimentation as a Service (EaaS) offering for innovation focused around urban data. In the same way our local authorities currently offer waste collection and road management as a service to citizens, we asked: could they expand their remit to offer experimentation with urban data as a service? EaaS was seen as a model where cities provide the resources for urban stakeholders (such as citizens, small businesses, grassroots, NGO's etc.) to develop, test and refine their new ideas at a small scale. These stakeholders become known as 'experimenters' when leveraging OrganiCity to develop their ideas. The service comprised of a series of innovative pillars as follows:

- Systematic experimentation: a framework of tools that enables easily testing ideas through a process that encourages evaluation of results at a small scale before iteration and expansion.

\footnotetext{
${ }^{1}$ www.organicity.eu - European Union Horizon 2020 grant 645198.
} 
- Co-creation: collaboratively defining how the service and the ideas experimented evolve with people at the core.

- Federated ethics and privacy: protection of users and data subjects (those individuals the data refers to) defined by default end to end throughout the service.

- Management of intellectual property rights: a legal framework that protects those collaborating in the innovation process.

In a city context, experimentation (the process of developing and testing out new ideas or approaches) is usually carried out in response to urban challenges such as in mobility, waste, wellbeing, sustainability etc. Experimenting in a city means ideas can be formulated, prototyped, tested and observed in a real-life urban setting. By doing short sprints of experimentation at a small, local scale, one can quickly learn what works and what doesn't. When something does work, it can be iterated and scaled up to a wider area or another city. When it doesn't work, this can be ascertained quickly, saving time and money by avoiding implementing unfeasible proposed solutions.

The OrganiCity EaaS facility can be seen as a toolbox, encompassing both organisational, technical (Digital Platform), funding \& guidance support mechanisms for enabling urban data and IoT experimentation that address pressing urban challenges. Pressing urban challenges are seen as those acknowledged by councils and citizens as requiring ameliorating actions. The urban data at the heart of the project was defined as data generated in a town or city region that can be measured - such as traffic levels, footfall, green spaces or even tweets. Questions motivating the OrganiCity project included:

- Why is citizen co-creation important for cities?

- What is the value-add proposition that sustains citizen engagement?

- What are the key challenges in the marketplace to which OrganiCity is responding?

Living Lab principles lay at the heart of OrganiCity, both in terms of its development as an EaaS facility and in its offering to experimenters. As such, experimenters were expected to integrate co-creation concepts and methodologies from problem formulation to their proposed solutions, as a condition of their use of the EaaS facility. By ensuring all relevant stakeholders were involved in the development of the solutions OrganiCity supports, it was anticipated that stakeholders such as city officials and citizens were more invested in the experimenters proposed solutions. In turn, such solutions would be better 'fit-for-purpose', have increased likelihood of adoption and have greater potential impact in terms of sustaining and scaling solutions.

Our approach involved iterating these principles during two phases of experimentation between 2016 to 2018. The 43 'experimenter' projects involved multiple stakeholders including citizens, entrepreneurs, small and medium businesses, grassroots organisations, research institutions, charities and local communities, and were rolled-out in 14 cities across Europe. An overview of the technical platform is described by Gutiérrez et al. (2016), discussions on the design of the platform are provided by Amaxilatis et al. (2018), whilst McLoughlin et al. (2019) examine differing urban data focused approaches taken by experimenters, and factors in sustaining their commercial solutions.

\section{Experimentation as a Service (EaaS)}

As an experiment itself, OrganiCity followed a human centric design approach with roughly half the team developing a technical platform and half focusing on the service design of the facility. The core team at the start of the project consisted of partners from three cities: Aarhus, London and Santander. This helped us to understand and evaluate the different cultural considerations when implementing EaaS across differing city environments. In the second phase of experimentation, additional cities where invited to become "OrganiCities" and implement the EaaS in their local context. A total of 14 cities participated in the experiments during the lifetime of the project.

The project was divided into 2 phases of open calls: In each phase, 'experimenters' were invited to submit proposals to experiment in the city. OrganiCity then assessed, selected, funded and supported experiments. Finally, OrganiCity assessed and iterated it's offering and showcased its impact through including the experimenters' journey and their engagement with respective communities. Each phase began by working with city councils and citizens to devise city challenges and target audiences for the open calls. Following the launch of open calls, clinics were organised in each city to launch the open calls and engage with prospective experimenters. Upon assessment and selection of experimenter proposals, OrganiCity support ensued whereby experiments were supported financially, technically and operationally for up to 6 months in duration.

Both during and after each experiment phase, the EaaS facilities design and technical requirements were evaluated and iterated with citizens, experimenters and OrganiCity. During the experimenters' journeys, OrganiCity took steps to ensure the impact and sustainability of the experiments (Mcloughlin et al., 2019) through creating external awareness, encouraging sustainability plans, hosting experimenter networking events, and encouraging those in phase 1 to advance their solution development by applying for phase 2 . Observations from the first open call led to the identification of 4 key service pillars which in turn were used as a focus for analysis in the second phase of implementation. The 4 service pillars which provide the foundations for this work are outlined below.

\subsection{Service Pillar 1 - Systematic Experimentation}

A foundational concept for OrganiCity was to create an interoperable framework of services to simplify experimentation for both technical and non-technical consumers of an EaaS facility. The goal was to allow the testing of ideas easily through a process that encourages 
evaluation of results at a small scale before iteration and expansion.

The technical architecture of OrganiCity (OrganiCity, 2018-A) highlights how the OrganiCity digital platform offered to experimenters exposes a set of functionalities and services through a series of API's and components. The OrganiCity digital platform includes:

- $\mathrm{AAA}-$ accounts management

- APIs - asset discovery, asset annotation, permissions, data sources

- $\quad$ Services - Urban Data Observatory (UDO) (UI to data and data annotation), experimenter portal, community management, facility manager

- Tools - scenario portal, TSmarT (IoT kit), TinkerSpace (mobile app), Smartphone experimentation framework, SensiNact (data stream IDE), web socket processing library

- Data assets - federated assets from several cities both historical and real time.

The digital platform provided the suite of tools or IT capabilities required for co-creation and validation of ideas under real world environments for both advanced developers and experimenters with a medium level of technical literacy. The goal was to enable experimenters to evaluate ideas quickly. In this respect, by providing technical capabilities and related documentation to experimenters such as grassroots or start-ups, the rationale was that such IT capabilities would ease and quicken the process of getting an idea off the ground and running. For instance, a start-up may need to invest much time and effort in developing in-house IT capabilities and knowhow to develop proposed urban data focused solutions. OrganiCity documentation targeted both expert and beginner audiences; GitHub access was given to all the source code, and 'how to' videos on using OrganiCity technology related services provided the guidance and support needed to engage a broad community of experimenters.

Creating standard interoperable access to data sources via the Urban Data Observatory and the API's supported the goal of making open access to the tools being used to build city services. This open access also provided for additional shared services such as reputation and data annotation modules supporting qualitative data annotation of data by citizens. It was anticipated that this meant experimenters would not need to have complete end to end technical capabilities and skills to develop and run a proposed urban data solution. Instead they could use existing OrganiCity digital capabilities via the digital platform to facilitate prototyping their idea and instead focus on other core areas of development.

The particular design of the OrganiCity digital platform also simplified its replication of services to other cities. For example, in the second phase of experimentation, the city of Lisbon federated their own instance of an OrganiCity server using a Docker container supporting virtualisation of the service. The simplicity of this federated approach demonstrated the potential to provide white label "OrganiCity in a box" servers.

\subsection{Service Pillar 2 - Co-Creation}

The human centred, design led approach to developing the citizen centric service required a number of different disciplines on the development team. The diverse skill sets included service designers, content leads, communications team, developers, technologists etc. By encouraging everyone in the city to become active participants in developing solutions increases the likelihood of participation. This makes the process more resilient since co-creation ensures a focus on the issues that matter to those involved and they become invested in making sure the experiment has the best possible outcome.

Workshops and clinics were run as part of the process of understanding pressing challenges in the various cities at the start of each phase of OrganiCity. These were a crucial component of the co-creation aspect of the project. By bringing together citizens, community groups, local authorities etc. to discuss issues, and the potential for urban data enabled services; more refined city challenges were formulated for the open calls and expectations could be managed both in terms of the kind of challenges citizens wanted solved and maturity of technology available in solving them.

A key observation from the project was the beneficial role the co-creation approach can play to support and facilitate civic engagement. A set of engagement principles provided the foundations to connect with different stakeholders from across the city. The seven principles (OrganiCity, 2018-B) are:

- Empower adjacent communities and champion advocates

- Design for trust, especially around change

- Facilitate personal \& community ownership

- Debate and co-create across comfort zones

- Use challenge areas as catalysts for innovation

- Respect the value of venue: the right space at the right time

- Provide a clear journey for participation and value visibility

The engagement principles were tested and adapted to the local contexts of Aarhus, London and Santander to gain insight into variations across the cities. Both online and offline approaches were implemented prior to challenges, during the call periods and once the experimental phase was in progress. The cocreation process also enabled collaboration between potential experimenters meeting through the open call process and provided a neutral platform for city officials to engage with people who may not have traditionally engaged with local officials.

\subsection{Service Pillar 3 - Federated Ethics and Privacy}

A key goal for OrganiCity was to establish methods for ensuring every experimenter would consider the ethics and privacy implications of their projects. Recognising the 
potential for such an approach to create "friction" in the innovation process and potentially slowing down progress, a framework was designed to protect those involved in the experimentation service. The project was completed before the implementation of the European General Data Protection Regulation (GDPR), but the OrganiCity architecture principles and guidelines directly anticipated this framework. OrganiCity did indeed explicitly go beyond the static regulation of the use and re-use of data by making the entire data ecosystem infrastructure transparent and inspectable in a more symmetrical way by any member of a city or community.

During both phases of experimentation those who applied to take part were required to complete an ethics and privacy checklist which in turn was used as part of the selection process, was reviewed by two assessors and was required prior to signing of the Experimenters Agreement. During experimentation the experimenters' approach to tackling ethics and privacy issues were monitored through a reporting mechanism and mentoring from a dedicated resource on the project.

\subsection{Service Pillar 4 - Management of Liability and Intellectual Property Rights}

At the outset of the project it was understood the EaaS would need to consider a legal framework that protects those collaborating in the innovation process. A legal framework was established in the Experimenters Agreement which provided a service level agreement addressing intellectual property and liability.

Often the legal considerations of experimenting in the public realm can create friction that overcomes trying to get an innovative idea off the ground. The framework developed provided support for those who may not have access to legal advice through providing standardised documentation providing boilerplate content and a checklist of items requiring review. The main principles of the agreement included:

- Ownership of results

- IPR of OrganiCity Facility

- Copyrights, processes and branding

- Access rights

- Service guarantees

All experimenters were required to sign the agreement at the outset to build trust between different parties involved in the process and included a user-friendly summary at the front of the legally-binding legal document to help make the necessary legal processes more inclusive. The OrganiCity brand now resides with the global Open \& Agile Smart Cities (OASC) ${ }^{2}$ network of cities and communities which represents the public demand-side in the emerging smart city market of data and services. The OrganiCity platform represented the first reference implementation of the so-called OASC Minimal Interoperability Mechanisms (MIMs). The ownership to assets and innovations remains a key focus area, and it is currently part of the OASC Ecosystem Transaction Management MIM.

\section{Observations from the experiments}

In this section we present key observations based on data collection involving in-depth interviews with experimenters, experimenter documents, as well as publicly available blogs and materials. We followed a grounded theory approach in collecting and analysing data. Primary data was collected using open-ended in-depth interviews, and thematically coded and analysed using MaxQdA qualitative software package. During interviews with experimenters, responses were elicited on the anticipated, current and future value they attributed to OrganiCity, as well as challenges encountered when experimenting, and issues associated with experimenting using city assets.

\subsection{Value-Proposition}

Upon analysis of experimenters involved with OrganiCity. We can surmise that the core value proposition of the OrganiCity EaaS approach can potentially be two-fold; i.e. enabling and supporting:

- $\quad$ experiments that are needed to achieve an evidence base (i.e. generate datasets) for addressing critical city issues

- experiments to develop and evaluate an urban data/IoT prototype solution for realising a business or organisation's (e.g. social enterprise) product or service.

In the first instance, we found several experimenters in phase 1 such as grassroots movements or social activists became involved with OrganiCity to gather an evidence base of urban data with which to achieve specific civic actions. In phase 2 funding experimenters was more contingent on them sustaining solutions beyond the funding period of OrganiCity. In this respect, OrganiCity as EaaS facility could potentially be envisioned as a city authority capability for enabling and supporting bottom-up citizen engagement around urban data. Existing initiatives such as 'DoCoMoMo' (DoCoMoMo 2019), 'Code for All' (CFA, 2019) and MySociety (MySociety, 2019) etc. offer space and/or support for citizens to get involved in social innovation at the grassroots level. OrganiCity conceived as a city authority provided EaaS facility, could potentially offer a new form of funding and support for citizen activism, thus promoting Deep Democratisation (Feenberg, 2012; Johnson, 2014) in the production of the smart city. Furthermore, OrganiCity could provide both inside and outside facing services in this regard. In other words, OrganiCity could also be leveraged by local authority staff/units and city officials to 'experiment' in gathering an evidence base of urban data or prototyping new solutions in order to understand and tackle specific city challenges. 
In the second instance, the predominant mode of experimenter involvement in OrganiCity from social enterprises (e.g. Space Engagers) to start-ups (e.g. Green Roof Monitoring) etc. was to develop (an) IS/IT hardware/software artefact(s) ranging from IoT to Smartphone app etc. in order to generate and/or exploit and/or visualise, and/or deliver insights around urban data within or across various verticals from mobility to environment to social welfare etc. OrganiCity offered the needed support (funding and/or digital platform and/or guidance etc) and favourable approach (i.e. Co-creation, realworld experimentation and federated cities) which was seen by many experimenters as ideal in the earlier stages of experimentation. Again, we should iterate that such a value proposition of OrganiCity as a city authority EaaS facility could support experiments initiated from both inside and outside a city authority's organisational boundaries.

\subsection{Becoming Involved with OrganiCity}

When asked to discuss why experimenters became involved in OrganiCity, the most common response or rational for becoming involved was 'funding', which closely related (by number of responses) to the second most common response; the 'opportunity to experiment' or 'trial' and develop a solution which was otherwise not feasible. As most experimenters were small start-up's or SME's, funding was seen as a crucial mechanism to either start or progress their company. Several experimenters also referred to the proposition of OrganiCity as being aligned with the early stage of their development cycle, providing the space to explore and co-create a solution.

A number of responses referred to the co-creation approach of OrganiCity in terms of engaging public-private stakeholders as being valuable. In this respect the supportive role for cocreation activities involving city and city council stakeholders attracted these experimenters to the OrganiCity proposition. However, a number of other themes emerged in response to this question. Individual experimenters referred to the opportunity to 'build external awareness', 'develop their networks', 'carry out multi-city experiments' and access 'technical expertise/capabilities'. For example, in the latter regard, several experimenters referred to the value of the Urban Data Observatory (OrganiCity, 2018-C), with 'DoItKits' relying on the Urban Data Observatory as a core component of the experiment and solution in terms of visualising output for end users. As interviews progressed, these latter themes formed a significant part of the value experimenters subsequently attributed to OrganiCity.

\subsection{Experimenters recognising the challenges with experimentation and planning accordingly}

Developing for the first time novel urban data/IoT solutions under the support of OrganiCity, led to significant learnings by experimenters in ensuring co-creation focused experiments that best addressed city challenges. The inter-related issues of; time, budget, communication, stakeholder-recruitment, access, as well as suitable co-creation methods employed were key resource constraints. In terms of time, themes emerged in overcoming time constraints of experimentation whilst staying within budget and ensuring co-creation activities could be organised given availability of stakeholders, such as city officials and companies.

Closely related to the issue of time, was available budget, whereby a challenge was ensuring suitable co-creation activities could be organised given significant financial constraints of small start-ups and grass-roots organisations. In some cases, experimenters cited this constraint as influencing and prioritising the type of co-creation activities undertaken. For some, ensuring recruitment of stakeholders (particularly end-users) formed a key consideration. For example, FSTR refined how they recruited students for their co-creation workshops to include intermediaries and social-media platforms based on initial poor response.

In terms of communication, experimenter's ability to best engage with end-users or other stakeholders through cocreation activities was seen as a challenge they worked to overcome, such as the case with Empati who initially designed mock-up's of their solution to engage citizens on intended prototype deployment. Access, both in terms of (a) access to facilities or (b) city assets, and in (c) ensuring access by endusers with special needs, resulted in key learnings which experimenters felt could be applied to their future experimentation. For example, given time and financial resource constraints, Wayfindr organised a two day co-creation activity with visually impaired users in order to evaluate and iterate an audio navigation solution for the London underground. Wayfindr experienced challenges in securing both the location with TFL, as well as ensuring their visually impaired users were suitably catered for. A challenge they successfully overcame through careful planning facilitated through the co-creation process.

Finally, several experimenters referred to evolving and even developing new co-creation methods through the process of experimentation as needs arose. Whilst some experimenters referred to the challenge of ensuring co-creation activities were sufficient in scope and number, others referred to unexpected co-creation activities emerging. For example, Leapcraft referred to unexpected extensive dialogue with partner bus companies in negotiating access, installation and experimentation of air pollution sensors across the three European cities involved. Although many of the experimenters had previously been involved in formal or informal co-creation activities, the specific nature of co-creation focused experimentation in the context of urban IoT and data solutions led to unforeseen challenges being negotiated. Furthermore, running multi-city experiments created additional unforeseen challenges in terms of issues such as (1) experiment planning, (2) distribution of available resources, (3) access to gatekeepers, (4) end-user visualisation and (5) adjustment to specific city typologies, politics, economics, cultural and legal circumstances.

\subsection{Developing techniques for deployment on assets}

Many of the experimenters developed or iterated on techniques to deploy solutions on city assets. Ten example issues 
overcome by some of the experimenters deploying their own IoT devices as part of their experimentation are as follows: Both 'Airpublic' and 'Leapcraft' developed novel methods for capturing accurate air pollution data on (1) moving assets / vehicles across the city. In the case of 'Airpublic', power requirements for the devices meant opting for vehicles instead of bicycle transport. Furthermore, 'Airpublic' validated data by setting pass-through geo-points whereby readings from different journeys across a single geo-point helped (2) validate device readings. In the case of 'Leapcraft', multiple IoT devices mounted on vehicles ensured readings from each helped validate each other. Furthermore, both companies experienced GPS and connectivity lapses across cities due to differing city typologies (e.g. London compared to Santander), requiring that multiple route journeys helped address gaps in data accuracy and data transfer. The experimenters recalled specific techniques developed in (3) positioning and installation on vehicles (including wiring, cabling), as well as overcoming challenges in prototype design for vehicle installation. In the case of 'Airpublic', this required design of a pump system for ensuring air pass-through and air pressure are optimal for (4) ensuring accurate readings on moving assets. In the case of 'Empati', a soft-sensor device was designed in a way to (5) blend into existing natural environments whilst being robust enough to (6) withstand physical elements and human use, whilst 'Wayfindr' leveraged the experimentation opportunity to refine techniques in Bluetooth beacon positioning in order to ensure (7) accurate and smooth data capture and transfer for end-user audio navigation. Finally, 'DoItKits' iterated IoT software and hardware to accurately capture water pollution data, dealing with specific (8) access, (9) power and (10) connectivity issues when wishing to deploy in London river sites. In sum, experimenting with OrganiCity provided valuable opportunities to develop and refine techniques in addressing the challenge of deploying devices in stationary and moving assets and settings across the respective cities.

\subsection{Experienced benefits attributed to OrganiCity}

Beyond the initial lure of OrganiCity in terms of funding and early stage experimentation etc., benefits which experimenters attributed to being involved in OrganiCity focused on a number of key themes in relation the 4 key service pillars of experimentation, co-creation, ethics and intellectual property. Many experimenters referred to extending their networks, including engagement with other experimenters to develop potential partnerships. Experimenters engaging in multi-city experimenters achieved significant learnings comparing differing city contexts (e.g. political, city typology, cultural, behaviour) including future challenges to scaling their solutions. The OrganiCity requirement to integrate co-creation activities from problem ideation to testing and evaluation resulted in experimenters putting in place a series of engagements from involving citizens, businesses, city councils and even technical expert stakeholders which experimenters reported as benefiting proposed solutions.

In terms of value from experimentation, the OrganiCity emphasis on a co-creation approach to solution development led to activities that had a substantive impact on experimental outcomes, including user insight led refinement of urban data visualisation (Public Like Displays; Leapcraft), the crowdsourcing of geo based datasets (Tranquil City), user centred design of a soft-sensing IoT probe (Empati), Generation of 'chatbot' app and qualitative dataset (Colour In City), a mobile air pollution IoT proof of concept (Airpublic), hackathon development of API and ITU audio navigation standard (Wayfindr) and an adjusted carpooling service offering based on local city typology and culture (FSTR).

\subsection{Future value attributed to OrganiCity}

The most common perceived future value of OrganiCity concerned the multi-city or federated nature of OrganiCity. Both single-city and multi-city experimenters referred to the future value of OrganiCity in supporting the 'testing' or 'trialling' of solutions in further cities beyond the open-calls. The second most common response related to the need for greater and better ease and availability of urban datasets to enrich experiments and the solutions being developed. Thirdly, improved capability of datasets through the UDO and Asset Discovery was cited in terms of supporting historical data, greater volumes of data, and data formats. The sophistication of advanced features with which to analyse, overlay and visualise data through the UDO was seen as adding value to OrganiCity's offering.

Several experimenters also referred to OrganiCity's value in promoting and building experimenters external awareness, as well as the networking opportunity of OrganiCity in learning about other experiments, developing social capital and potential future partners. In the former respect, as well as helping promote experimenters, OrganiCity was seen as potentially lending access and credibility to experimenters' efforts to co-create with stakeholders, by building trust, credibility and awareness as an outlet of innovation. In the latter regard, experimenters referred to the 'OrganiCity network'. For example, one experimenter referred to the potential for OrganiCity to become, 'a European access point for innovation' in the urban data and IoT space.

Other themes to emerge related to OrganiCity as an 'access point' or 'information intermediary' to the city, as an initiative in promoting a standardised and structured approach to data and API's across cities, with the need to recruit an end-user base for future experimenters being cited. The value of OrganiCity becoming open to the supply or access of data by experimenters beyond open call access was also cited, as well as the value in OrganiCity's Digital platform becoming open source so as to develop its technical capability and user base.

\subsection{Observations beyond the experimenters}

In addition to data collection from those experimenting, we have been continually soliciting feedback from those working in or with those in public office (e.g. members of the Smart London Board, OASC members etc). As part of these efforts, a range of city stakeholders in London were interviewed in 2017. The major themes generated from analysing these 
interviews which have broader applicability on the value of OrganiCity for public office are described below:

- Mechanism to exploit urban data stores: Many cities are starting to create "open datasets" to share publicly available data (e.g. London Data Store https://data.london.gov.uk/ and the newly formed London Office of Technology Innovation). As the technical challenges to reduce the friction in creating those services is overcome, the attention is now turning to the mechanisms needed for exploiting such data. Services such as those provided in OrganiCity potentially forms one such mechanism.

- Engaging Innovation on urban data: In extension to making data available, a clear priority for city hall concerns encouraging engagement around innovation and urban data. OrganiCity facilitated the inclusion of a broader audience in the use of a city's digital assets.

- Mechanism to renew city services: Comparing EaaS to related Living $\mathrm{Lab}$ initiatives, examples of successful Living Lab set-ups concerned cities who used Living Labs as a mechanism to renew, develop or extend their own services.

- Standardised tool-set for experimentation: The 'standardised way of working' or 'Toolbox' approach to experimentation is seen as something valuable that OrganiCity is promoting. The principle of designing in interoperability from the start was an attractor for the potential for sharing resources across urban organisations.

- Framework for challenge based experimentation: OrganiCity tools and approach for co-creation involving both social and technical approaches, which start from defining city challenges, helps build ownership of change and enables change-makers to make change.

- Enabler of commercial success: The success stories of experimenters arising out of OrganiCity will largely define how cities view the commercial offering of OrganiCity.

\section{Lessons Learnt}

Through the process of running the EaaS offering in many diverse city types across Europe, some key lessons or takeaways emerged. The 4 service pillars described in this paper demonstrated that:

- Tools need maintenance. The technical platform developed by several of the OrganiCity consortium partners was central in helping cities offer innovative civic services. But, more crucially, they need the investment in maintenance and ongoing operational support as part of their design. Maintaining a productive suite of services used by and with the community requires continued and iterative development.

- EaaS requires financial and human capital. Both in supporting technical operation or in managing the engagement of citizens in the formative challenge stages or 'production' phase of the experiments. When discussing the longer-term sustainability of the EaaS facility with city officials the human (staff) cost or running such a service is almost always greater than the technical cost of installing and hosting the technical infrastructure.

- Governance is different but similar. Ethics, privacy and IPR were designed into the EaaS platform from the outset in response to the need to create trustworthy civic tech and to support the commercial requirements of the start-ups and SME's trying to create new businesses. In defining the frameworks and legal guidelines to support these bureaucratic necessities a set of blueprints were established to facilitate participation by the OrganiCity Cities and sped up the process of deployment and experimentation.

Whilst the OrganiCity project was a significant investment in testing the EaaS approach in many European cities there are inevitably some limitations to highlight. This project focused on fourteen organisations developing a prototype platform over a three-year period with 43 experimenters and 14 cities engaging in that journey. This meant that the process of service design for how the cities would engage in the experimentation open calls was in continual development. In parallel the systems and services were in continual evolution requiring a level of expectation management between the technical and non-technical members of the team in how the EaaS would be described to the community user base (OrganiCity, 2018-D).

Key challenges moving forward include measuring and communicating the value of the OrganiCity EaaS approach in terms beyond efficiency savings or cost reductions in city hall services but in terms of the social benefit of engagement with citizens and access to resources to those who are typically digitally excluded. Any benchmarking against other methods for driving innovation such as innovation funds, lottery funding or incubator programmes would need to look at both the social and economic measures of success.

In summary, a key strength of the project was the development of an Experimentation as a Service blueprint that cities can use to engage citizens in co-developing civic tech innovations that utilise the emerging digital infrastructure being developed in our urban environment. We found that it was useful for democratically sourcing, testing and scaling up innovations emerging from a broad cross section of civil society and an analysis of the experimenter projects highlighted the importance of shared infrastructure making a difference in bringing their ideas to market. 
Whilst the OrganiCity project completed in 2018 the ideas and learnings have been foundational in the development of the global Open \& Agile Smart Cities non-profit network (OASC 2018) and the practical implementations continue to develop in the SynchroniCity project (SynchroniCity, 2018).

\section{Acknowledgements}

This work was supported by the OrganiCity research project funded by the European Union, under the grant agreement No. 645198 of the Horizon 2020 research and innovation program.

\section{References}

Amaxilatis, D. et al. (2018) Advancing Experimentation-as-aService Through Urban IoT Experiments, in IEEE Internet of Things Journal. doi: 10.1109/JIOT.2018.2871766

Chesbrough, H., Vanhaverbeke, W., \& West, J. (Eds.). (2006) Open innovation: Researching a new paradigm. Oxford University Press on Demand.

CFL (2019) Creating fair and open digital societies. https://codeforall.org/ (accessed 19/12/18)

Coenen, T., van der Graaf, S., \& Walravens, N. (2014) Firing up the city-a smart city living lab methodology. Interdisciplinary Studies Journal, 3(4), 118.

Curley, M., \& Salmelin, B. (2017) Open innovation 2.0: the new mode of digital innovation for prosperity and sustainability. Springer.

DoCoMoMo (2019) https://www.docomomo.com/index/ (accessed 19/12/18)

Dutilleul, B., Birrer, F. A., \& Mensink, W. (2010) Unpacking European Living Labs: Analysing Innovation's Social Dimensions. Central European journal of public policy, 4(1). 60-85.

Feenberg, A. (2012) Questioning Technology. London: Routledge. https://doi.org/10.4324/9780203022313

GLA (2016) Data for London - A City Data Strategy, Greater London Authority. https://data.london.gov.uk/dataset/data-forlondon-a-city-data-strategy (accessed 19/12/18)

Gutiérrez, V., Theodoridis, E., Mylonas, G., Shi, F., Adeel, U., Diez, L., ... \& Muñoz, L. (2016) Co-creating the cities of the future. Sensors, 16(11), 1971. doi:10.3390/s16111971

Johnston, M. (2014) Deep democratization and the control of corruption. In Corruption, contention and reform: the power of deep democratization. Cambridge University Press.

McLoughlin, S., Maccani, G., Prendergast, D., \& Donnellan, B. (2018) Living Labs: A Bibliometric Analysis. In Proceedings of the 51st Hawaii International Conference on System Sciences. In https://doi.org/10.24251/HICSS.2018.563.

Mcloughlin, S., Puvvala, A., Maccani, G., \& Donnellan, B. (2019) A Framework for understanding \& classifying Urban Data Business Models. In Proceedings of the 52nd Hawaii International Conference on System Sciences.
MySociety (2019) Technology, research and data that help people to be active citizens. https://www.mysociety.org/about/ (accessed 19/12/18)

OASC (2018) https://oascities.org/ (accessed 19/12/18)

OrganiCity (2018-A) Overview of Technical Architecture. https://docs.OrganiCity.eu/ (accessed 19/12/18)

OrganiCity (2018-B) Guide to engage with people across the city. http://OrganiCity.eu/wpcontent/uploads/2017/06/Guideto-engage-with-people-across-the-city.pdf (accessed 19/12/18)

OrganiCity (2018-C) Urban Data Observatory. https://docs.OrganiCity.eu/UrbanDataObservatory/ (accessed 19/12/18)

OrganiCity (2018-D) OrganiCity Playbook. http://OrganiCity.eu/wp-content/uploads/2018/06/OrganiCityPlaybook_2018-1.pdf (accessed 19/12/18)Schuurman, D., De

Schuurman, D., Marez, L. and Ballon, P. (2015) Living Labs: a systematic literature review. Open Living Lab Days 2015

SynchroniCity (2018) https://synchronicity-iot.eu/ (accessed 19/12/18)

TfL (2017) TfL's free open data boosts London's economy: Transport for London. https://tfl.gov.uk/info-for/media/pressreleases/2017/october/tfl-s-free-open-data-boosts-london-seconomy (accessed 19/12/18)

Wilson, D. (2018) Do living labs facilitate city-wide collaboration and innovation? Smart London Board. https://medium.com/@SmartLondon/do-living-labs-facilitatecity-wide-collaboration-and-innovation-b112f67a0475 (accessed 19/12/18) 